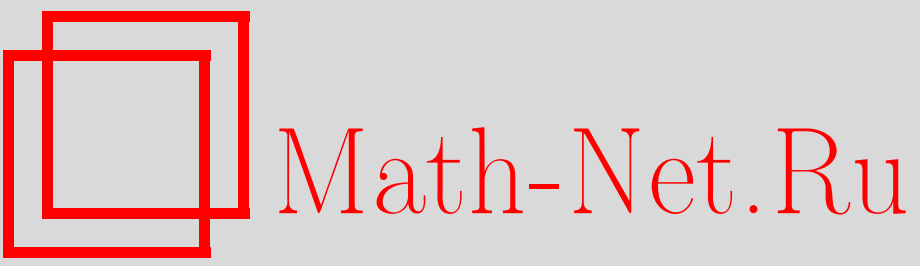

Л. Д. Пустыльников, О представлении дзета-функции Римана в критической полосе через бесконечное произведение матриц второго порядка и об одной динамической системе, УМН, 2004, том 59, выпуск 4, 197-198

DOI: https://doi.org/10.4213/rm765

Использование Общероссийского математического портала Math-Net.Ru подразумевает, что вы прочитали и согласны с пользовательским соглашением

http://www.mathnet.ru/rus/agreement

Параметры загрузки:

IP : 54.162 .85 .209

26 апреля 2023 г., $15: 46: 12$ 


\section{О ПРЕДСТАВЛЕНИИ ДЗЕТА-ФУНКЦИИ РИМАНА В КРИТИЧЕСКОЙ ПОЛОСЕ ЧЕРЕЗ БЕСКОНЕЧНОЕ ПРОИЗВЕДЕНИЕ МАТРИЦ ВТОРОГО ПОРЯДКА И ОБ ОДНОЙ ДИНАМИЧЕСКОЙ СИСТЕМЕ}

\section{Л. Д. Пустыльников}

Хорошо известно, что дзета-функция Римана $\zeta(s)$ представляется в виде бесконечного произведения Эйлера только в полуплоскости $\operatorname{Re} s>1(\operatorname{Re}-$ вещественная часть $s)$. В настоящей работе доказано, что в критической полосе $0<\operatorname{Re} s<1$ функция $\zeta(s)$ представляется через бесконечное произведение конкретных матриц второго порядка (теорема 1). Это представление впервые было получено в [1], однако существенная часть доказательства, которая сформулирована и доказана ниже в лемме, не была до сих пор опубликована. Эта лемма - не тривиальная, так как ее утверждение несправедливо, например, при $s=0$ и при любом натуральном числе $k \geqslant 2$, а доказателшство существенно использует условие $0<\operatorname{Re} s<1$. Теорема 1 вместе с основным результатом работы [1] позволяет построить динамическую систему, которая оказьвается связанной с гипотезой Римана о нулях так, что каждому комплексному нулю функции $\zeta(s)$, не лежащему на прямой $\operatorname{Re} s=1 / 2$, соответствует периодическая траектория второго порядка специального вида (теорема 2 и ее следствие).

Лемма. При целом $k \geqslant 2$ рассмотрим функции

$$
h_{k}(s)=1 / k^{s}-\left(k^{1-s}-(k-1)^{1-s}\right) /(1-s) .
$$

Тогда в области $0<\operatorname{Re} s<1$ справедливы неравенства $h_{k}(s) \neq 0, k=2,3, \ldots$.

ДокАЗАТЕльство. Предположим, что $h_{k}(s)=0,0<\operatorname{Re} s<1$. Тогда в силу (1) получаем: $1-s=k\left(1-(1-1 / k)^{1-s}\right)$,

$$
\operatorname{Re}(1-s)=\operatorname{Re}\left(k\left(1-(1-1 / k)^{1-s}\right)\right) .
$$

Пусть $s=\sigma+i t$, где $\sigma$ и $t$-вещественные числа, $0<\sigma<1$. Имеем:

$$
(1-1 / k)^{1-s}=(1-1 / k)^{1-\sigma}(\cos \theta+i \sin \theta),
$$

где $\theta$ - вешественное число. Поэтому

$$
\beta_{s} \stackrel{\text { def }}{=} \operatorname{Re}\left(k\left(1-(1-1 / k)^{1-s}\right)\right)=k\left(1-(1-1 / k)^{1-\sigma} \cos \theta\right) \text {. }
$$

Рассмотрим два случая, случай 1$): \cos \theta \leqslant 0$, и случай 2$): \cos \theta>0$. В первом случае в силу неравенств $0<\operatorname{Re} s<1$ при $k \geqslant 2$ получаем неравенство $\operatorname{Re}(1-s)=1-\sigma<k \leqslant \beta_{s}$, и, следовательно, в силу определения $\beta_{s}$ в (3) равенство (2) несправедливо. Во втором случае, используя неравенства $\cos \theta>0,0<\sigma<1$, имеем:

$$
0<(1-1 / k)^{1-\sigma} \cos \theta \leqslant(1-1 / k)^{1-\sigma}=1-(1-\sigma) / k-\sum_{\nu=2}^{\infty} \gamma_{\nu} / k^{\nu},
$$

где $\gamma_{\nu}>0, \nu=2,3, \ldots,-$ константы, не зависящие от $k$. Подставляя (4) в (3), получаем неравенство $\beta_{s} \geqslant k\left((1-\sigma) / k+\sum_{\nu=2}^{\infty} \gamma_{\nu} / k^{\nu}\right)>1-\sigma$, которое также противоречит (2). Лемма доказана.

Лемма позволяет дать следующее определение.

Работа выполнена при частичной поддержке Российского фонда фундаментальных исследований (грант № 02-01-01067). 
ОПРЕДЕЛЕниЕ 1 . При $k=1,2, \ldots$ в области $0<\operatorname{Re} s<1$ рассмотрим функции $p_{k}(s)=$ $h_{k}(s) / h_{k-1}(s)$, где $h_{1}(s)=(s-1)^{-1}, h_{0}(s)=1$ и при целом $k \geqslant 2$ функции $h_{k}(s)$ введены в $(1)$, и определим матрицы

зависящие от $s$.

$$
Q_{k}=Q_{k}(s)=\left(\begin{array}{cc}
0 & 1 \\
-p_{k}(s) & -1-p_{k}(s)
\end{array}\right)
$$

Tеорема 1. Бесконечные произведения $Q_{\infty}^{\prime}(s)=\lim _{k \rightarrow \infty} Q_{2 k}(s) Q_{2 k-1}(s) \cdots Q_{1}(s)$ u $Q_{\infty}^{\prime \prime}(s)=\lim _{k \rightarrow \infty} Q_{2 k-1}(s) Q_{2 k-2}(s) \cdots Q_{1}(s)$ определень в области $0<\operatorname{Re} s<1$ u имеют следующий вид:

$$
Q_{\infty}^{\prime}(s)=\left(\begin{array}{cc}
-\zeta(s)+1 & -\zeta(s) \\
\zeta(s)-1 & \zeta(s)
\end{array}\right), \quad Q_{\infty}^{\prime \prime}(s)=\left(\begin{array}{cc}
\zeta(s)-1 & \zeta(s) \\
-\zeta(s)+1 & -\zeta(s)
\end{array}\right)
$$

Доказательство теоремы 1 содержится в доказательстве теоремы 2 из работы [1].

Обозначим через $\Pi=\{s: 0<\operatorname{Re} s<1\}$ критическую полосу, а через $l$ гильбертово пространство, элементами которого являются односторонние последователшности $x=\left(x_{1}, x_{2}, \ldots\right)$ комплексных чисел, удовлетворяющие условию $\|x\| \stackrel{d e f}{=} \sum_{\nu=1}^{\infty}\left|x_{\nu}\right|^{2}<\infty$.

Введем оператор $A(s)$, зависящий от комплексного числа $s \in$ П и действующий на $l$ следующим образом: если $x=\left(x_{1}, x_{2}, \ldots\right) \in l$, то $A(s) x=x^{\prime}=\left(x_{1}^{\prime}, x_{2}^{\prime}, \ldots\right)$, где $x_{k}^{\prime}=\sum_{j=1}^{\infty} a_{k j}(s) x_{j}$,

$$
a_{k j}(s)= \begin{cases}1, & \text { если } k-j=-1, \\ p_{k}(s), & \text { если } 0 \leqslant k-j \leqslant 1, \\ 0, & \text { если }|k-j|>1,\end{cases}
$$

$p_{k}(s)$ - функции, введенные в определении $1, k=1,2, \ldots$.

ОПредЕлЕниЕ 2. Введем пространство $\Omega=\Pi \times l \times l$, являющееся прямшім произведением $\Pi, l$ и $l$, и преобразование $T$ пространства $\Omega$ такое, что если $(s, x, y) \in \Omega(s \in \Pi, x \in l, y \in l)$, то $T(s, x, y)=\left(s^{\prime}, x^{\prime}, y^{\prime}\right)$, где $s^{\prime}=1-s, x^{\prime}=A\left(s^{\prime}\right) y, y^{\prime}=A(s) x$.

Теорема 2. Функция $\zeta(s)$ тогда и только тогда имеет нуль $s_{*} \in \Pi\left(\zeta\left(s_{*}\right)=0\right)$, удовлетворяющий условию $\operatorname{Re} s_{*} \neq 1 / 2$, когда существует точка $(s *, e, \delta) \in \Omega(e \in l$, $\delta \in l)$ maкая, что $\|e\|+\|\delta\| \neq 0$, и если $\left(s_{*}^{\prime}, e^{\prime}, \delta^{\prime}\right)=T\left(s_{*}, e, \delta\right)$, mo $e^{\prime}=-\delta, \delta^{\prime}=-e$.

ДокАЗАТЕльство. В силу определения 2 преобразования $T$, без ограничения общности достаточно рассмотреть случай $1 / 2<\operatorname{Re} s_{*}<1$. Если $\zeta\left(s_{*}\right)=0$, то в качестве $\delta$ возьмем последовательность, состоящую из одних нулей, а в качестве $e$ возьмем собственньй вектор оператора $A\left(s_{*}\right)$ с собственньм значением $\lambda=-1$, которьй существует в силу теоремы 1 из работы [1].

СлеДСтвиЕ. $E c л и \zeta\left(s_{*}\right)=0, s_{*} \in \Pi, \operatorname{Re} s_{*} \neq 1 / 2$, то отображение $T^{2}$ имеет неподвижную точку $\left(s_{*}, e, \delta\right) \in \Omega$ такую, что $\|e\|+\|\delta\| \neq 0: T^{2}\left(s_{*}, e, \delta\right)=\left(s_{*}, e, \delta\right)$.

\section{СПИСОК ЛИТЕРАТУРЫ}

[1] Л. Д. Пустыльников // УМН. 1991. Т. 46. № 2. С. 227-228. 